\title{
Rethinking The Law School. Education, Research, Outreach and Governance, de Carel \\ Stolker, Cambridge University Press, 2014
}

Antonio Ponce Rojo

Se trata de una obra extremadamente interesante, estructurada con base en 12 Capítulos, perfectamente equilibrados, a través de los cuales se analizan las escuelas de derecho en todo el mundo, sus funciones, las responsabilidades que la sociedad les ha encomendado y las expectativas que se pueden tener a propósito de su labor a mediano y largo plazo, dadas las circunstancias actuales de nuestro mundo inmerso en una educación globalizada.

En el primer capítulo, StoIker, actualmente rector de la Leiden University en los Países Bajos, analiza a las escuelas de derecho en Estados Unidos, el Reino Unido, Europa, Asia y en América Latina, sus características y las variaciones en tamaños, filosofías subyacentes y modelos educativos que están detrás de ellas. Estas fascinantes instituciones educativas han cargado durante si- glos la responsabilidad de educar, no solamente a los abogados y fiscales del mundo, sino a reyes, príncipes, presidentes, jueces, notarios, gerentes de empresas, banqueros entre otros, lo que nos da fácilmente la idea del gran peso de la encomienda social que sobre los hombros cargan estas escuelas. A lo largo de las páginas de este apartado, Stolker aborda las características de las escuelas de derecho que las distinguen de las escuelas del resto de profesiones y disciplinas; características que parecen ir incluso en contra de la enorme carga que pesa sobre sus hombros: una aparente tensión entre práctica legal y la investigación académica legal, una baja proporción de doctores en derecho como docentes, e incluso, en el caso de las escuelas que cuentan con programas de posgrado en derecho o sus especialidades, una baja proporción de tesis sobre temas centrales del derecho, siendo todos estos, los puntos que necesariamente deben estar en las agendas de desarrollo a mediano y largo plazo, de las universidades e institutos que las albergan. El propio 
Stolker narra cómo ha sido acusado de negativo en extremo cuando se refiere a las escuelas de derecho en el mundo, pero su lógica es aplastante: es necesario conocer la situación actual con honestidad y a profundidad, para poder plantear los retos y oportunidades.

En el Segundo y el Tercer Capítulo Stolker analiza primero, los retos estratégicos de las escuelas de leyes y después la búsqueda de identidad, ello con base en las características planteadas en el apartado anterior. Habla sobre puntos nodales en la planeación estratégica de estas instituciones: el reto de la aplicación del modelo de triple hélice, la lucha actual entre la masificación, la privatización, la diversificación y la atención de las necesidades del mercado, que en algunos casos es tomado como criterio principal para definir su pertinencia; aborda asimismo, el problema de la autonomía universitaria y la regulación del estado, la globalización e internacionalización, así como las presiones por la búsqueda de un alto nivel de calidad y competitividad certificados. En lo referente a la búsqueda de la identidad, el autor aborda la discusión frecuente acerca del lugar de las leyes en las universidades, las leyes como disciplina de estudio y como tema de investigación, incluyendo también una discusión alterna acerca de la necesidad o no de colaborar con otras disciplinas y ciencias, y de la misma manera, de la urgencia de apoyarse en ellas.

En los Capítulos Cuarto, Quinto y Sexto, Stolker analiza los elementos relacionados con la educación de los estudiantes de leyes, las pedagogías que imperan y la pertinencia de las becas y otros elementos de soporte a los estudiantes en su proceso de formación. Si ha quedado claro que las escuelas de leyes son diferentes al resto de las escuelas, los estudiantes igualmente difieren y las condiciones incluso, son también especiales, entonces se requieren pedagogías especiales y en ello descansa mucho de la misión de quienes diseñan los programas y las rutas académicas. Aborda en estos capítulos, algunos elementos de la Escuela de Derecho de Leiden, de la cual Stolker fue director hasta 2012, antes de llegar a ser rector de toda la Universidad.

En los Capítulos Séptimo, Octavo y Noveno, Stolker analiza los productos e impactos de las escuelas de leyes. Va desde las formas de publicación que son características en el derecho y sus disciplinas afines, hasta los impactos, primero a niveles social y económico y luego, a escala personal. El autor plantea la necesidad de voltear hacia una tercera misión de las universidades, después de la docencia y 
la investigación, sobre todo en momentos en donde los soportes gubernamentales se han convertido en todo el mundo en un elemento fundamental para el funcionamiento institucional. Esta tercer misión de la que habla Stolker, es la retribución a la sociedad de la inversión que ésta ha realizado en la educación y reconoce que aún cuando las escuelas de derecho en el mundo impactan a través de su principal producto, es decir, los abogados, es necesario ir más allá. Esta tercer misión, va más allá de la función sustantiva de "Extensión" que se reconoce en las universidades en América Latina, es llevada incluso más allá del contacto inicial con la sociedad, cuando se habla del compromiso de transparencia y de rendición de cuentas que necesariamente se adquiere al ser un elemento fundamental de ésta, pero también cuando se considera que se le ha encomendado dar respuesta a los grandes retos mundiales:

“... Las universidades, con sus brillantes científicos y académicos, cada vez son más requeridas para buscar soluciones a los grandes retos mundiales [...] no son más aquellas torres de marfil, sino instituciones cuyo alcance, visibilidad e involucramiento son ahora su orden cotidiano..." (p. 264-265).

En este mismo tenor, Stolker refiere el término de "Univerciudades" acuñado en 2007 por Bo Larsson, como una manera de dar cuenta de esta tercer misión de las Universidades a la que las escuelas de derecho en el mundo tienen mucho que aportar (p. 279).

Asimismo, en el Capítulo Noveno, el autor trata dos elementos fundamentales en la formación en leyes: la creatividad y el liderazgo. Habla de cómo las escuelas de derecho deben propiciar tres elementos básicos para todo líder de un grupo creativo: desarrollar ideas, ayudar a estructurar esas ideas y promover resultados (p. 345)

En la parte final del libro, los Capítulos Décimo, Undécimo y Duodécimo, Stolker trata temas relacionados con la trascendencia de las escuelas de derecho y su agenda a mediano y largo plazo. Habla de la integridad científica y académica, como elementos ideales en el proceso de formación de los nuevos abogados. Cierra la obra con los retos que las escuelas de leyes tienen enfrente: sacar provecho a aquello que las hace diferentes, trayendo el mundo a sus aulas y propiciando aquellos elementos que permiten que se eche mano de todos los recursos disponibles gracias a los avances tecnológicos, incluso, promoviendo la remoción de inercias, pero de manera crea- 
tiva; la promoción de la colaboración en todos los niveles y escalas, procurando con ello una diversificación incluso, en los modos de concebir los modelos educativos que subyacen a las instituciones tarea que impacta directamente a los académicos y sus formas de trabajo, pero también y sobre todo a las instituciones y sus maneras de impulsar el trabajo académico, ya que como Stolker dice citando a Boulton "cambiar una universidad es como tratar de mover una tumba, los que están adentro no te van a ayudar..." (p. 380).

En suma, repensar las escuelas de derecho, desde la perspectiva del autor, es una ambición que implica el reconocimiento de la necesidad de colaboración y el esfuerzo por fortalecer al Derecho como disciplina. La colaboración conllevará a todos aquellos elementos que se marcan como indispensables para el mejoramiento de la calidad: la movilidad, la internacionalización, la profesionalización, entre otros; mientras que el fortalecimiento del Derecho como disciplina llevará a el aporte hacia la tercer misión que la sociedad ha encomendado a las instituciones educativas y en donde las escuelas de derecho tienen una función básica.

Se trata así, de una obra que desde mi punto de vista debe ser considerada en la planeación estratégica de las escuelas de derecho y revisada por todos aquellos que tenemos una encomienda en el proceso de formación de los expertos del mañana. 


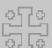

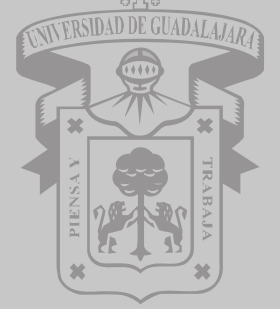

JURISPRUDENCIA DE

LA SUPREMA CORTE

DE JUSTICIA DE LA NACIÓN

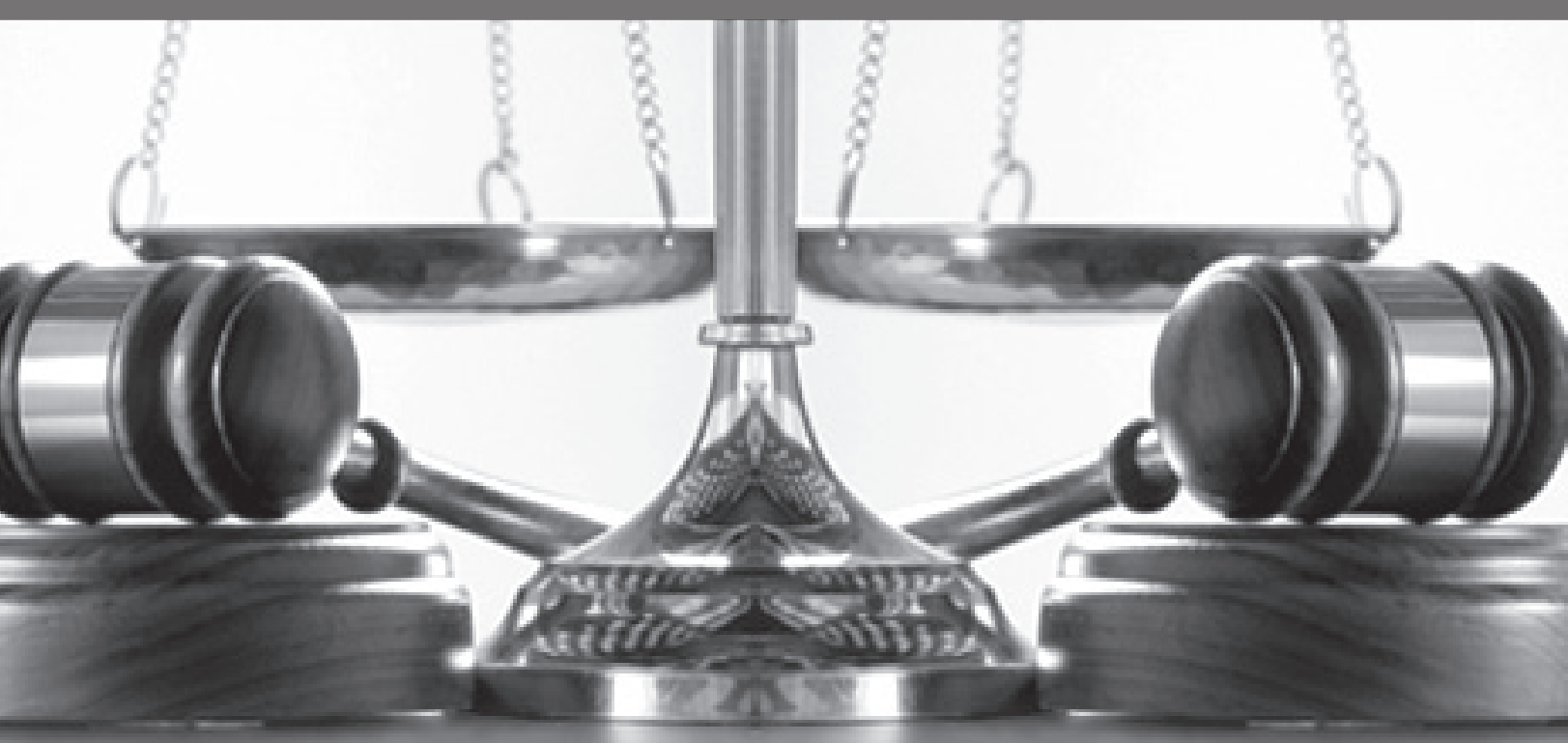




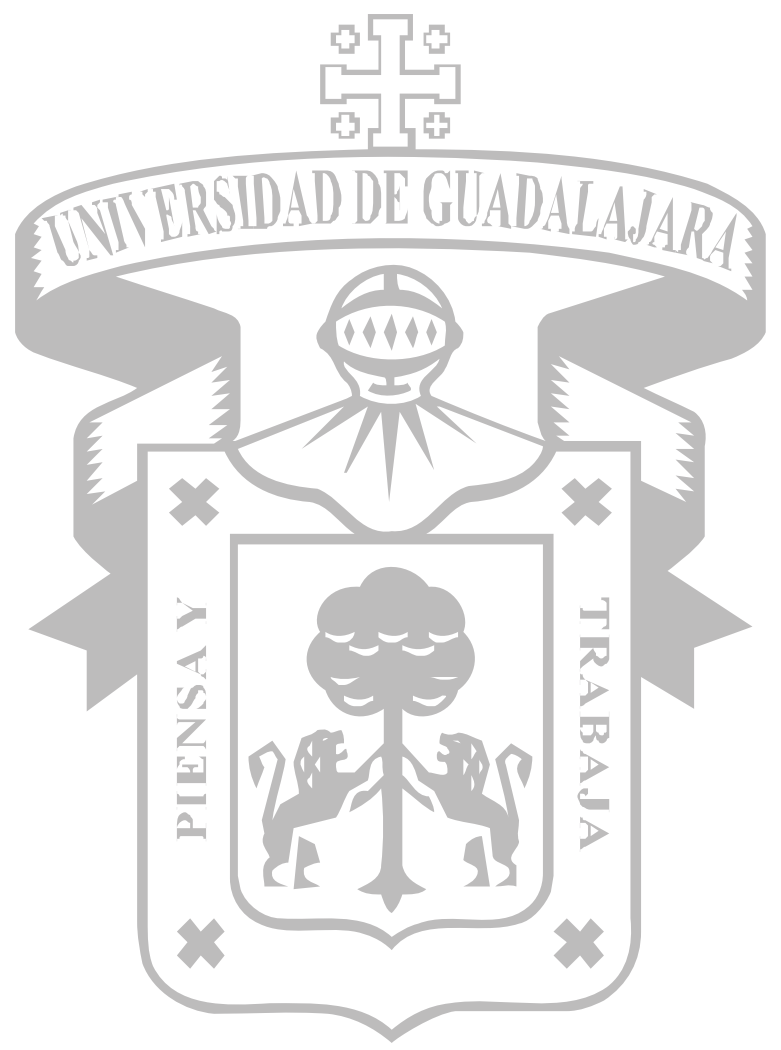

